\title{
A resistant case of neurocystercercosis
}

\author{
M.S. Chong, C.P. Hawkins, G.C. Cook, C.H. Hawkes and R.S. Kocen
}

The National Hospital for Neurology and Neurosurgery, Queen Square, London WC2, UK

\begin{abstract}
Summary: A case of neurocystercercosis resistant to treatment with repeated courses of albendazole and praziquantel (even in combination), leading to deterioration in clinical condition is described.
\end{abstract}

\section{Introduction}

Neurocystercercosis is a very important cause of epilepsy in South America, and is also a significant problem in Asia and Africa. ${ }^{1}$ A prompt response to praziquantel (or albendazole) can usually be anticipated. ${ }^{1-3}$ We present a patient whose condition progressed despite repeated courses of chemotherapy.

\section{Case report}

A 38 year old Indian housewife had two grand mal seizures at the age of 10 years and was treated with anticonvulsants for 18 months. She came to England at the age of 20 and remained well until 1973 when cervical and mediastinal tuberculous adenitis was diagnosed (on biopsy). She received a course of isoniazid and ethambutol for 1 year. Further grand mal seizures began in July 1988; phenytoin was commenced. Five months later she had right-sided focal fits; a CT-scan showed enhancing lesions in both cerebral cortices. A further course of antituberculous therapy was started but was discontinued on account of hepatotoxicity. Chest and thigh radiographs showed calcified lesions. Immunofluorescent antibody test for cystercercosis was positive ( 1 in 80 ), and she was given a 14-day course of praziquantel ( $50 \mathrm{mg} / \mathrm{kg}$ daily). In August 1988 , she noted persistent pruritus ani and bilateral facial twitching; there were no more generalized seizures. A further course of praziquantel was given. One year later she was readmitted to hospital with epilepsy partialis continua, right hemiparesis and dysphasia. An MRI scan showed extensive oedema of both cerebral hemispheres with multiple regions of high signal. Reactivation of neuro-

Correspondence: M.S. Chong, B.Sc., M.R.C.P., Department of Anatomy and Developmental Biology, University College London, Gower Street, London WC1E 6BT, UK.

Accepted: 5 February 1991 cystercercosis was suspected. She had a 30-day course of praziquantel $(2.5 \mathrm{~g})$ and albendazole (400 mg) daily, with corticosteroid cover; she made a good recovery. By March 1990, she complained of episodes of 'pressure sensation' within her head lasting minutes, associated with visual blurring and tinnitus. Following a further fit 3 months later which seemed to relieve her symptoms, she was noted to be drowsy and have a poor memory. Eventually, she was readmitted after a severe bout of vomiting and 'collapse'. A CT-scan showed oedema in the right cerebral hemisphere with multiple enhancing lesions. On examination, she had bilateral papilloedema. The remaining neurological examination, including tests of higher mental function, was normal. There were no other abnormal physical findings. Immunofluorescent antibody test for cystercercosis was again positive ( 1 in 80 ) but there was no evidence of cysts within the ventricular system (CT and MRI scans). Cerebral biopsy (at three sites within the right parietal region) was carried out; chronic inflammation with gliosis, but no evidence of granulomata or eosinophils, was present. She was commenced on a course of praziquantel and albendazole (for 60 days) and is currently well.

\section{Discussion}

The case illustrates the typical pattern of parenchymal central nervous system disease resulting from neurocystercercosis; focal and grand mal seizures with transient focal deficits and raised intracranial pressure. ${ }^{1}$ This form of neurocystercercosis is usually amenable to treatment with praziquantel or albendazole. This woman appears to have refractory form of disease that has progressed in spite of repeated courses of appropriate chemotherapy (even in combination).

Tuberculomas, hydatidosis, coenuriasis, sarcoidosis and primary and secondary tumours ${ }^{4}$ seem unlikely. Serology for hydatid and cryptococcosis 
was negative and brain biopsy did not show any granulomata. The presence of calcified lesions in muscle, and partial response to treatment with praziquantel and albendazole (which was associated with disappearance of her neurological signs) leads us to conclude that neurocystercercosis is the

\section{References}

1. Cook, G.C. Parasitic Diseases in Clinical Practice. SpringerVerlag, London, 1990, pp. 191-206.

2. Vasconcelos, D., Cruz-Segura, H., Mateos-Gomez, H. \& Zenteno Alanis, $H$. Selective indications for the use of praziquantel in the treatment of brain cystercercosis. $J$ Neurol Neurosurg Psychiatry 1987, 50: 383-388. correct diagnosis.

Although considered a readily treatable condition $^{1-3}$ this case proved to be relatively refractory to treatment. This emphasizes the requirement for a continued search for more effective chemotherapy and even more importantly, prevention.

3. Escobedo, F., Penagos, P., Rodriguez, J. \& Sotelo, J. Albendazole therapy for neurocystercercosis. Arch Intern Med 1987, 147: $738-741$.

4. Case records of The Massachussets General Hospital (Case 20-1990). N Engl J Med 1990, 322: 1446-1458. 